\title{
TEST FUNCTION SPACE FOR WICK POWER SERIES
}

\author{
A. G. Smirnov ${ }^{1}$, M. A. Soloviev"
}

\begin{abstract}
We derive a criterion that is convenient for applications and exactly characterizes the test function space on which the operator realization of a given series of Wick powers of a free field is possible. The suggested derivation does not use the assumption that the metric of the state space is positive and can therefore be used in a gauge theory. It is based on the systematic use of the analytic properties of the Hilbert majorant of the indefinite metric and on the application of a suitable theorem on the unconditional convergence of series of boundary values of analytic functions.
\end{abstract}

\section{Introduction}

We consider convergence conditions for infinite series of the form

$$
\sum_{k=0}^{\infty} d_{k}: \phi^{k}:(x)
$$

with respect to the Wick powers of a free field $\phi(x)$. Jaffe [1] first performed an analysis of this kind in determining the Borchers class of a massive free field in the two-dimensional space-time. He showed that the normally ordered entire functions of exponential type for this field are well defined as operator-valued tempered distributions satisfying all Wightman axioms. Subsequent works by Jaffe and some other authors showed that Wick series that are ultradistributions and hyperfunctions should also be included in the Borchers class and that it is natural to consider even more singular generalized functions in extending the axiomatic approach to nonlocal fields. Rieckers [2] showed that for any Wick series of a massive free field in a space-time of dimension

\footnotetext{
${ }^{1}$ Lebedev Physics Institute, RAS, Moscow, Russia.
} 
$\mathrm{d} \geq 2$, there is a suitable Gelfand-Shilov space $S^{\beta}$ such that this series is convergent under averaging with test functions belonging to $S^{\beta}$. The generalization of the technique in [1, 2], which is essentially based on the positive definiteness of the two-point function $\Delta^{+}(x ; m)$, to fields with an indefinite metric is far from obvious, although precisely fields with an indefinite metric are of particular interest because the exact solutions of some simple gauge models, including the Schwinger model, can be expressed in terms of the normal exponentials of such fields. The problem of an adequate choice of test functions when the positivity condition is violated was raised by Wightman [3] and was analyzed in [4, 5, 6] for the examples of a massless scalar two-dimensional field and a dipole ghost field. An important observation in [4, 5, 6] is that the operator realization of the field (目) in the case of an indefinite metric is only possible under some conditions on the test functions that are more restrictive than those under which the Wightman functions of this field, which are calculated using the Wick theorem, are defined. The direct analysis of series convergence in the momentum representation by analogy with Rieckers' analysis is extremely laborious. In [7], a different approach was suggested based on using the analytic properties of the Hilbert majorant of the indefinite metric in the coordinate representation and investigating the convergence (in an appropriate topology) of series of analytic functions instead of series of distributions that serve as the boundary values of these functions. We show that a criterion can be thus elaborated that solves the problem in the general form and permits easily finding the test function space for any Wick series (11) with given coefficients proceeding from the infrared and ultraviolet behavior of the two-point function of the free field $\phi(x)$.

We consider the generalized Gelfand-Shilov spaces $S_{a}^{b}$ and $S^{b}[8]$ and use the results in 9] that permit operating with functionals in the dual spaces $S_{a}^{\prime b}$ and $S^{\prime b}$ as simply as with the usual Schwartz distributions. The definitions needed for the proofs are given below. Here, we only mention that the indices $a$ and $b$, which were number sequences in the original statement in [8], can now be identified with the corresponding indicator functions characterizing the decrease at infinity of the test functions in $S_{a}^{b}$ and of their Fourier transforms and that the elements of $S^{b}$ decrease faster than any power of $1 /|x|$ as $|x| \rightarrow \infty$. The elements of the most frequently used spaces $S_{\alpha}^{\beta}$ in this scale have an exponential decrease of the order $1 / \alpha$ in the coordinate representation and of the order 
$1 / \beta$ in the momentum representation.

In Sec. 2, we present the necessary facts related to quantization with an indefinite metric and specify the precise meaning of the convergence of the series (11) in the pseudoHilbert state space. We prove the key convergence theorem for series of boundary values of analytic functions in Sec. 4. An extension lemma for functionals that permits proving this theorem rather simply is derived in Sec. 3. We obtain the ultimate general convergence criterion for the Wick series of a free field in Sec. 5. In Sec. 6, we give applications to the case of a positive metric that strengthen the results in [2]. We consider an example of entire functions of the two-dimensional massless free field that have a finite order of growth in Sec. 7. In this example, the singularities are logarithmic, and the use of the generalized spaces $S_{a}^{b}$ instead of $S_{\alpha}^{\beta}$ permits characterizing the related test function space much more precisely. Concluding remarks are presented in Sec. 8 .

\section{Graph limits of Wick power series}

Under the positivity condition, the operator realization of a free field $\phi(x)$ is reconstructed from its two-point function $w\left(x-x^{\prime}\right)$ up to a unitary equivalence. The major difficulty in generalizing the reconstruction theorem to the indefinite-metric case lies in properly relating to $w$ a distribution $w_{\text {maj }}\left(x, x^{\prime}\right)$ of a positive type that determines the Hilbert topology with respect to which the completion gives the state space $\mathcal{H}$. The solution of this problem is described in [10, 11], and we take the resulting mathematical structure as the starting point. The space $\mathcal{H}$ is endowed by two sesquilinear forms $\langle\cdot, \cdot\rangle$ and $(\cdot, \cdot)$ that are determined by $w$ and $w_{\text {maj }}$ and are related by the formula

$$
\langle\Phi, \Psi\rangle=(\Phi, \theta \Psi), \quad \Phi, \Psi \in \mathcal{H}
$$

where $\theta$ is an involutory and Hermitian operator with respect to the positive form $(\cdot, \cdot)$ (it is known as the Krein operator). This pseudo-Hilbert structure is compatible with the Fock structure by which the space $\mathcal{H}$ is also endowed because we deal with a free theory. The brackets $\langle\cdot, \cdot\rangle$ and $(\cdot, \cdot)$ are expressed in terms of $w$ and $w_{\text {maj }}$ at the one-particle level by the formulas

$$
\left\langle\phi(f) \Psi_{0}, \phi(g) \Psi_{0}\right\rangle=\int \bar{f}(x) w\left(x-x^{\prime}\right) g\left(x^{\prime}\right) \mathrm{d} x \mathrm{~d} x^{\prime}
$$




$$
\left(\phi(f) \Psi_{0}, \phi(g) \Psi_{0}\right)=\int \bar{f}(x) w_{\text {maj }}\left(x, x^{\prime}\right) g\left(x^{\prime}\right) \mathrm{d} x \mathrm{~d} x^{\prime},
$$

where $\Psi_{0}$ is the vacuum state and $f, g$ are test functions belonging to the Schwartz space. The compatibility with the Fock structure means that the relations

$$
\begin{gathered}
\left\langle: \phi\left(f_{1}\right) \ldots \phi\left(f_{m}\right): \Psi_{0},: \phi\left(g_{1}\right) \ldots \phi\left(g_{n}\right): \Psi_{0}\right\rangle=\delta_{m n} \sum_{\pi} \prod_{j}\left\langle\phi\left(f_{j}\right) \Psi_{0}, \phi\left(g_{\pi(j)}\right) \Psi_{0}\right\rangle \\
\left(: \phi\left(f_{1}\right) \ldots \phi\left(f_{m}\right): \Psi_{0},: \phi\left(g_{1}\right) \ldots \phi\left(g_{n}\right): \Psi_{0}\right)=\delta_{m n} \sum_{\pi} \prod_{j}\left(\phi\left(f_{j}\right) \Psi_{0}, \phi\left(g_{\pi(j)}\right) \Psi_{0}\right),
\end{gathered}
$$

hold, where the summation extends over all permutations of indices.

In what follows, we use the properties that the majorant $w_{\text {maj }}$ must have by its sense and that can be easily verified in every specific case. Namely, we assume that $w_{\text {maj }}$ as well as $w$ belongs to the class $S^{\prime}$ of tempered distributions and inherits the spectral properties of $w$ in the sense that the function

$$
\hat{w}_{\text {maj }}\left(p, p^{\prime}\right)=\int e^{i p x+i p^{\prime} x^{\prime}} w_{\text {maj }}\left(x, x^{\prime}\right) \mathrm{d} x \mathrm{~d} x^{\prime}
$$

satisfies the condition

$$
\operatorname{supp} \hat{w}_{\text {maj }}\left(p, p^{\prime}\right) \subset \overline{\mathbb{V}}_{+} \times \overline{\mathbb{V}}_{-},
$$

where $\mathbb{V}_{+}$and $\mathbb{V}_{-}$are the upper and lower light cones and the bar symbolizes the closure. We stress that the majorant need not be translation invariant, and we therefore do not impose the condition $p+p^{\prime}=0$. In terms of the coordinate representation, the above assumption means that $w_{\text {maj }}$ is the boundary value of an analytic function $\mathbf{w}_{\text {maj }}\left(z, z^{\prime}\right)$ that is holomorphic in the tubular domain $\left\{z, z^{\prime}: y=\operatorname{Im} z \in \mathbb{V}_{-}, y^{\prime}=\operatorname{Im} z^{\prime} \in \mathbb{V}_{+}\right\}$ and satisfies the condition

$$
\left|\mathbf{w}_{\text {maj }}\left(z, z^{\prime}\right)\right| \leq C(V)\left(1+|z|+\left|z^{\prime}\right|\right)^{\omega}\left(|y|+\left|y^{\prime}\right|\right)^{-\sigma} \quad\left(y, y^{\prime} \in V\right),
$$

where $V$ is an arbitrary compact 1 cone in $\mathbb{V}_{-} \times \mathbb{V}_{+}$and the numbers $\omega \geq 0$ and $\sigma \geq 0$ do not depend on $V$.

The convergence of the series (1) as well as the existence of the Wick monomials themselves, which are formally defined by the relation

$$
: \phi^{k}:(x)=\lim _{x_{1}, \ldots, x_{k} \rightarrow x}: \phi\left(x_{1}\right) \ldots \phi\left(x_{k}\right):,
$$

\footnotetext{
${ }^{2}$ The compactness means that the inclusion $\bar{V} \backslash\{0\} \subset \mathbb{V}_{-} \times \mathbb{V}_{+}$holds. Here and henceforth, the norm $|\cdot|$ is assumed to be Euclidean.
} 
should be established in the topology defined by the scalar product $(\cdot, \cdot)$. A more exact statement of the definition is the following. Let $f \in S\left(\mathbb{R}^{\mathrm{d}}\right)$, where $\mathrm{d}$ is the space-time dimension. We take an arbitrary $\delta$-shaped sequence $\delta_{\nu}(x)$ (i.e., convergent to the Dirac delta function in $S^{\prime}$ ) of nonnegative test functions that also belong to $S\left(\mathbb{R}^{\mathrm{d}}\right)$ and consider the sequence of operators

$$
\varphi_{\nu}(k, f)=\int: \phi\left(x_{1}\right) \ldots \phi\left(x_{k}\right): f\left(x_{1}\right) \prod_{j=2}^{k} \delta_{\nu}\left(x_{1}-x_{j}\right) \mathrm{d} x_{1} \ldots \mathrm{d} x_{k}
$$

with the domain $D_{0}$ composed of linear combinations of $\Psi_{0}$ and vectors of the form

$$
\Psi=\int: \phi\left(x_{1}\right) \ldots \phi\left(x_{n}\right): g\left(x_{1}, \ldots, x_{n}\right) \mathrm{d} x_{1} \ldots \mathrm{d} x_{n} \Psi_{0}, \quad g \in S\left(\mathbb{R}^{n \mathrm{~d}}\right), \quad n=1,2, \ldots
$$

It can be shown that the sequence (6) has a strong graph limit not depending on $\delta_{\nu}(x)$, which is precisely the limit denoted by $: \phi^{k}:(f)$. Its domain contains all vectors of the form

$$
\prod_{j=1}^{n}: \phi^{k_{j}}:\left(g_{j}\right) \Psi_{0}
$$

where $g_{j} \in S\left(\mathbb{R}^{\mathrm{d}}\right)$, whose linear span serves as the invariant domain of all monomials. Each monomial is a tempered operator distribution satisfying the Lorentz covariance, locality, and spectral conditions. It should be noted that the technique below permits substantially simplifying the routine derivation of these facts, but we do not dwell on this point.

We now introduce the notation

$$
\varphi_{N}(f)=\sum_{k \leq N} d_{k}: \phi^{k}:(f)
$$

and analyze the convergence conditions for the series (1). We first consider the action of the partial sums in (8) on the vacuum state. According to the definition of : $\phi^{k}$ : and formulas (3) and (4), we have

$$
\left\|\varphi_{N}(f) \Psi_{0}\right\|^{2}=\sum_{k \leq N}\left|d_{k}\right|^{2}\left\|: \phi^{k}:(f) \Psi_{0}\right\|^{2}=\sum_{k \leq N} k !\left|d_{k}\right|^{2} w_{\mathrm{maj}}^{k}(\bar{f} \otimes f),
$$

whence the effect of the absence of positivity is clearly seen, namely, the series in the right-hand side has the same structure as the series of distributions representing the two-point function of the field (1) with $w$ replaced by $w_{\text {maj }}$. For the operator realization 
of the field (11), its arbitrary repeated action on $\Psi_{0}$ must be defined. We indicate the domain of definition in $\mathcal{H}$ that is invariant not only with respect to this action but also with respect to the action of any field determined by a series with coefficients $d_{k}^{\prime}$ such that $\left|d_{k}^{\prime}\right| \leq C\left|d_{k}\right|$, where $C$ is a constant depending on the series. This condition establishes a preorder on the set of the series. We let $\triangleleft$ denote this preorder and say that the series with the coefficients $d_{k}^{\prime}$ is subordinate to the series with the coefficients $d_{k}$.

Lemma 1. Let $\phi$ be a neutral scalar free field acting in the pseudo-Hilbert space $\mathcal{H}$, let $\mathfrak{s}(\phi)$ be the series (11) in the Wick powers of this field with $d_{1} \neq 0$, let $E$ be a barrelled space of test functions that is dense in $S$, and let $D^{E}$ denote the subspace of $\mathcal{H}$ generated by $\Psi_{0}$ and by the vectors of the form $(\overline{7})$, where $g_{j} \in E$. If for every positive integer $n$ and for arbitrary sets of series $\mathfrak{s}^{(j)} \triangleleft \mathfrak{s}$ and functions $f_{j} \in E, 1 \leq j \leq n$, the sequence of vectors

$$
\Phi_{N}=\sum_{\substack{k_{j} \leq N \\ 1 \leq j \leq n}} d_{k_{1}}^{(1)} \ldots d_{k_{n}}^{(n)}: \phi^{k_{1}}:\left(f_{1}\right) \ldots: \phi^{k_{n}}:\left(f_{n}\right) \Psi_{0}, \quad N=1,2, \ldots
$$

where $d_{k_{j}}^{(j)}$ are the coefficients of $\mathfrak{s}^{(j)}$, converges in the norm of $\mathcal{H}$, then the series $\mathfrak{s}(\phi)$ with the domain $D^{E}$ converges in the sense of the strong graph limit to a field $\varphi$ that is an operator-valued generalized function over E. Moreover, any Wick series subordinate to $\mathfrak{s}$ is convergent, and all these fields have the common domain $D^{E}(\mathfrak{s})$, which is dense and invariant.

Proof. Let $\Psi_{N}, \Psi_{N}^{\prime} \in D^{E}, \Psi_{N} \rightarrow \Psi, \Psi_{N}^{\prime} \rightarrow \Psi, \varphi_{N}(f) \Psi_{N} \rightarrow \Phi$, and $\varphi_{N}(f) \Psi_{N}^{\prime} \rightarrow$ $\Phi^{\prime}$. By the definition of the strong graph limit [12], we should show that $\Phi=\Phi^{\prime}$. Let

$$
\Omega=: \phi\left(f_{1}\right) \ldots \phi\left(f_{n}\right): \Psi_{0}, \quad f_{j} \in E, \quad 1 \leq j \leq n
$$

It follows from the Wick theorem that

$$
\varphi_{N}(f)^{*} \mid D^{E}=\sum_{k \leq N} \bar{d}_{k}: \phi^{k}:(\bar{f})
$$

where the superscript $*$ symbolizes the pseudo-Hermitian conjugation. Since $d_{1} \neq 0$, the sequence $\varphi_{N}(f)^{*} \Omega$ is a finite linear combination of sequences (10) of particular form and consequently is convergent. Hence,

$$
\left\langle\Phi-\Phi^{\prime}, \Omega\right\rangle=\lim _{N \rightarrow \infty}\left\langle\varphi_{N}(f)\left(\Psi_{N}-\Psi_{N}^{\prime}\right), \Omega\right\rangle=\lim _{N \rightarrow \infty}\left\langle\Psi_{N}-\Psi_{N}^{\prime}, \varphi_{N}(f)^{*} \Omega\right\rangle=0
$$


and therefore the desired relation $\Phi=\Phi^{\prime}$ holds because the linear span of the vectors of the form (11) is dense in $\mathcal{H}$, which follows from the density of $E$ in $S$. To show this, suppose $\bar{\Omega}$ is a vector of the same form but with the test functions $g_{j} \in S$ and consider $f_{j \nu} \in E, f_{j \nu} \rightarrow g_{j}$ in $S$. We write $g=g_{1} \otimes \ldots \otimes g_{n}, f_{\nu}=f_{1 \nu} \otimes \ldots \otimes f_{n \nu}$ and $\Omega_{\nu}=: \phi\left(f_{1 \nu}\right) \ldots \phi\left(f_{n \nu}\right): \Psi_{0}$. Then

$$
\left\|\bar{\Omega}-\Omega_{\nu}\right\|^{2}=T_{n}\left(\left(\overline{f-f_{\nu}}\right) \otimes\left(f-f_{\nu}\right)\right)
$$

where

$$
T_{n}\left(x, x^{\prime}\right)=\sum_{\pi} \prod_{j=1}^{n} w_{\text {maj }}\left(x_{j}, x_{\pi(j)}^{\prime}\right) .
$$

according to (3) and (4). The membership relation $T_{n} \in S^{\prime}$ implies $\Omega_{\nu} \rightarrow \Omega$, and the abovementioned denseness property holds by virtue of the cyclicity of the vacuum.

Let $\varphi(f)$ denote the graph limit of the sequence (8). A pair $\{\Psi, \Phi\}$ belongs to the graph of this operator if there is a sequence $\Psi_{N} \in D^{E}$ such that $\Psi_{N} \rightarrow \Psi$ and $\varphi_{N}(f) \Psi_{N} \rightarrow \Phi$. The partial sums of any subordinate series $\mathfrak{s}^{(j)}(\phi)$ also have limits. The vectors $\prod_{1 \leq j \leq n} \varphi^{(j)}\left(f_{j}\right) \Psi_{0}$, where $f_{j} \in E$ and $n=1,2, \ldots$, are well defined, and the linear span of $\Psi_{0}$ and of all vectors of this form can be taken as the subspace $D^{E}(\mathfrak{s})$. This subspace is invariant and dense in $\mathcal{H}$. The role of the barrelledness condition for $E$ is that for barrelled spaces, the uniform boundedness principle holds and the pointwise convergence of the sequence of continuous linear mappings $f \rightarrow \varphi_{N}(f) \Psi_{N}$ from $E$ to $\mathcal{H}$ implies the continuity of the limit mapping $f \rightarrow \varphi(f) \Psi$ (see theorem III.4.6 in [13). As a consequence, $\langle\Phi, \varphi(f) \Psi\rangle$ is a generalized function over the space $E$ for any $\Phi, \Psi \in D^{E}(\mathfrak{s})$. The lemma is proved.

The expression for the $n$-point vacuum expectation value of the field $\varphi$ given by the Wick theorem is a power series in $n(n-1) / 2$ variables $w\left(x_{j}-x_{m}\right)$ and can be written as

$$
\left\langle\Psi_{0}, \varphi\left(x_{1}\right) \ldots \varphi\left(x_{n}\right) \Psi_{0}\right\rangle=\sum_{K} D_{K} W^{K}
$$

where $K$ is an integer-valued vector with nonnegative components $k_{j m}, 1 \leq j<m \leq n$, and

$$
W^{K} \stackrel{\text { def }}{=} \prod_{j<m} w\left(x_{j}-x_{m}\right)^{k_{j m}}
$$


The combinatorial analysis related to the Wick theorem shows [1] that

$$
D_{K}=\frac{\kappa !}{K !} \prod_{1 \leq j \leq n} d_{\kappa_{j}}
$$

where $\kappa_{j}=k_{1 j}+\ldots+k_{j-1, j}+k_{j, j+1}+\ldots+k_{j n}$ is the total number of pairings in the given term of the series that involve the argument $x_{j}$, and we follow the usual convention

$$
K !=\prod_{j<m} k_{j m} !, \quad \kappa !=\prod_{1 \leq j \leq n} \kappa_{j} ! .
$$

We use one more simple lemma to derive specific convergence conditions for the multi-index series whose partial sum is the vector (10).

Lemma 2. Let $I$ be a countable set of indices and let $\left\{\Phi_{k}\right\}_{k \in I}$ be a family of elements in the Hilbert space with the scalar product $(\cdot, \cdot)$. If the double number series $\sum_{k, l \in I}\left(\Phi_{k}, \Phi_{l}\right)$ is absolutely convergent, then the family $\left\{\Phi_{k}\right\}_{k \in I}$ is unconditionally summable.

Proof. By definition, unconditional summability means that for any one-to-one mapping $\nu \rightarrow k_{\nu}$ of the set of nonnegative integers $\mathbb{N}$ onto $I$, the series $\sum_{\nu \in \mathbb{N}} \Phi_{k_{\nu}}$ is convergent, i.e., for any $\epsilon>0$ there is an index $\lambda_{0}$ such that $\left\|\sum_{\lambda \leq \nu \leq \Lambda} \Phi_{k_{\nu}}\right\|<\epsilon$ for $\lambda_{0} \leq$ $\lambda \leq \Lambda$. Since the square of the norm involved does not exceed $\sum_{\lambda \leq \mu, \nu \leq \Lambda}\left|\left(\Phi_{k_{\mu}}, \Phi_{k_{\nu}}\right)\right|$, the assertion of the lemma follows from the elementary fact that a convergent series of positive numbers remains convergent and satisfies the Cauchy criterion for convergence under an arbitrary permutation of its terms. Lemma 2 is proved.

Thus, the assumptions of Lemma 1 hold if the number series

$$
\sum_{\substack{k_{1}, \ldots, k_{n} \\ l_{1}, \ldots, l_{n}}}\left(\prod_{1 \leq j \leq n} d_{k_{j}}: \phi^{k_{j}}:\left(f_{j}\right) \Psi_{0}, \prod_{1 \leq j \leq n} d_{l_{j}}: \phi^{l_{j}}:\left(f_{j}\right) \Psi_{0}\right),
$$

which represents $\left\|\prod_{1 \leq j \leq n} \varphi\left(f_{j}\right) \Psi_{0}\right\|^{2}$ in this case, is absolutely convergent for some $n$ and for all $f_{j} \in E$. In what follows, we keep to the argument indexing that corresponds to the formal representation of this expression in the form $\int\left(\varphi\left(x_{1}\right) \ldots \varphi\left(x_{n}\right) \Psi_{0}, \varphi\left(x_{n+1}\right) \ldots \varphi\left(x_{2 n}\right) \Psi_{0}\right) \overline{f_{1}\left(x_{1}\right) \ldots f_{n}\left(x_{n}\right)} f_{1}\left(x_{n+1}\right) \ldots f_{n}\left(x_{2 n}\right) \mathrm{d} x_{1} \ldots \mathrm{d} x_{2 n}$. Bringing each of the products of Wick powers to the totally normally ordered form (see Appendix $A$ in $[1]$ ) and then applying (团), we obtain

$$
\left\|\prod_{1 \leq j \leq n} \varphi\left(f_{j}\right) \Psi_{0}\right\|^{2}=\sum_{K} D_{K} W^{K}(\bar{f} \otimes f),
$$


where $f=f_{1} \otimes \ldots \otimes f_{n}$. This expression is similar to the formula (12) with the obvious change $n \rightarrow 2 n$ in the definition of multi-indices $K$ and $\kappa$. Moreover,

$$
D_{K}=(\kappa ! / K !) \prod_{1 \leq j \leq n} \bar{d}_{\kappa_{j}} \prod_{n \leq j \leq 2 n} d_{\kappa_{j}}
$$

A more important feature is that in this case we have

$$
W^{K}=\prod_{1 \leq j<m \leq n} w\left(x_{m}-x_{j}\right)^{k_{j m}} \prod_{n+1 \leq j<m \leq 2 n} w\left(x_{j}-x_{m}\right)^{k_{j m}} \prod_{\substack{1 \leq j \leq n \\ n+1 \leq m \leq 2 n}} w_{\text {maj }}\left(x_{j}, x_{m}\right)^{k_{j m}} .
$$

The problem has now been reduced to finding convergence conditions for a series of distributions of a special structure whose terms serve as boundary values of analytic functions in the same tubular domain. The major difficulty lies in determining the type of the singularity of the sum of the series if it is summable. The singularity of a functional is related to the behavior of its Fourier transform at infinity, and we characterize it by estimating the convolutions of the Fourier transform with rapidly decreasing test functions.

\section{$3 \quad$ Extension lemma}

Lemma 3. Let $u \in S_{b_{0}}^{\prime a}$ and $b(s) \leq b_{0}(s)$. If the convolutions of a functional $u$ with the functions $g \in S_{b_{0}, B}^{a, A}$ satisfy the inequality $B>0 \quad A>0$

$$
|(u * g)(p)| \leq C_{\epsilon, A}\|g\|_{A, B} b(\epsilon|p|)
$$

with an arbitrary $\epsilon>0$ for some $B>0$ and any $A>0$, then the functional $u$ has a unique continuous extension to the space $S_{b}^{a}$. If the family of functionals in $S_{b_{0}}^{\prime a}$ satisfies the uniform estimate (15), then the extended functionals form a bounded set in $S_{b}^{\prime a}$. Similarly, if $u \in S_{b_{0}}^{\prime}$ and the inequality

$$
|(u * g)(p)| \leq C_{\epsilon}\|g\|_{B, N} b(\epsilon|p|)
$$

with an arbitrary $\epsilon>0$ and some $N$ (which can depend on $\epsilon$ ) holds for some $B$ and

all $g \in S_{b_{0}, B}$, then the functional $u$ has a unique extension to an element of the space $S_{b}^{\prime}$, and extending the family of functionals results in a bounded set in the case of the uniform estimate. 
Lemmas of this type were used in [9], but here we give this refined statement suitable for our further aims. We stress that the indicator functions of these spaces possess some regularity properties according to their definition. In particular, they are convex and monotonically increasing beginning with unity, and therefore

$$
b\left(\left(s_{1}+s_{2}\right) / 2\right) \leq\left(b\left(s_{1}\right)+b\left(s_{2}\right)\right) / 2 \leq b\left(s_{1}\right) b\left(s_{2}\right) .
$$

Moreover, the inequality

$$
s b(s) \leq C b(\lambda s)
$$

with some constants $C$ and $\lambda$ must hold, which ensures the existence of the operation of multiplication by the independent variable. The two properties expressed by (17) and (18) are essential for deriving Lemma 3 , and the sequence $b_{0}$ must even satisfy the condition

$$
b_{0}^{2}(s) \leq C b_{0}(\lambda s)
$$

which is stronger than (18). Here, we only prove Lemma 3 for the case of functionals of the class $S_{b_{0}}^{\prime}$, which was not considered in [9]. We recall that $S_{b_{0}}$ is the union of the countably normed spaces $S_{b_{0}, B}$ and that the norm $\|g\|_{B, N}$ in (16) has the form

$$
\|g\|_{B, N}=\sup _{q} \max _{|\kappa| \leq N}\left|\partial^{\kappa} g(q)\right| b_{0}(|q| / B) .
$$

The desired extension $\tilde{u}$ can be defined by the formula

$$
(\tilde{u}, h)=\int\left(u, h_{0}(p-\cdot) h(\cdot)\right) \mathrm{d} p, \quad h \in S_{b},
$$

where $h_{0}$ is an arbitrary element in $S_{b_{0}, B / \lambda}$ with the property

$$
\int h_{0}(p) \mathrm{d} p=1
$$

The formula (16) implies the inequality

$$
\left|\left(u, h_{0}(p-\cdot) h(\cdot)\right)\right| \leq C_{\epsilon}\left\|g_{p}\right\|_{B, N} b(\epsilon|p|)
$$

where $g_{p}(q) \stackrel{\text { def }}{=} h_{0}(q) h(p-q)$. If $h \in S_{b, B_{1}}$, where $B_{1} \geq B$, then applying the Leibniz formula gives

$$
\left\|g_{p}\right\|_{B, N} \leq 2^{N}\left\|h_{0}\right\|_{B / \lambda, N}\|h\|_{B_{1}, N} \sup _{q} \frac{b_{0}(|q| / B)}{b_{0}(\lambda|q| / B) b\left(|p-q| / B_{1}\right)}
$$


Consecutively applying the property (19), the inequality $b(s) \leq b_{0}(s)$ and the formula (17) that the fraction in the right-hand side of (22) can be estimated from above by the function $C / b\left(|p| / 2 B_{1}\right)$. Substituting it in (21) and taking (18) into account, we see that the integrand in (20) decreases faster than polynomially as $|p| \rightarrow \infty$ and the integral specifies a linear functional on $S_{b}$, whose continuity is guaranteed by the presence of the factor $\|h\|_{B_{1}, N}$ in the right-hand side of (22). The constructed functional coincides with the original one on the functions $h \in S_{b_{0}}$ because in this case, the integral (20) exists not only for $u$ but also for any distribution in $S_{b_{0}}^{\prime}$. Consequently, the sequence of Riemann integral sums for $\int h_{0}(p-\cdot) h(\cdot) \mathrm{d} p$ is weakly Cauchy in the space $S_{b_{0}}$ and because this is a Montel space $f$, it converges in $S_{b_{0}}$ to an element that can only be $h$. This consideration also proves that $S_{b_{0}}$ is dense in $S_{b}$ and the extension is hence unique. If a family of functionals belonging to $S_{b_{0}}^{\prime}$ satisfies the uniform estimate (16), then the set of their extensions to $S_{b}^{\prime}$ is obviously weakly bounded, and because $S_{b}$ is a barrelled space, this set is also strongly bounded.

\section{Convergence conditions for series of boundary values of analytic functions}

As already mentioned, the distributions entering the multi-index series (13) are the boundary values of the holomorphic functions $\mathbf{v}_{K}$ in the same tubular domain. The base this domain is a convex cone $V$, and for any compact subset $Q \subset V$, the condition of polynomial boundedness

$$
\left|\mathbf{v}_{K}(x+i y)\right| \leq C(1+|x|)^{\omega} \quad(y \in Q),
$$

holds, where the constants $C$ and $\omega$ generally depend on $Q$. We first consider the series (13) on the spaces $S_{a}^{1}$, where the index 1 means that $\beta=1$, i.e., the indicator function $b(s)$ of these spaces is $e^{s}$. The dual spaces of $S_{a}^{1}$ consist of hyperfunctions with the order of growth $\sim a(|x|)$ at infinity. As is known, hyperfunctions provide the widest framework for constructing a local field theory. By definition, we have $S_{a}^{1}=\cup_{A, B>0} S_{a, A}^{1, B}$, where $S_{a, A}^{1, B}$ is the Banach space of functions which are analytic in the

\footnotetext{
"In [8], the term "perfect space" was used instead of "Montel space", which is now commonly accepted. See [13] for the properties of topological vector spaces we use here.
} 
domain $T_{B}=\{x+i y:|y|<1 / B\}$, continuous in its closure and have the finite norm

$$
\|f\|_{A, B}=\sup _{z \in T_{B}}|f(z)| a(|x| / A) .
$$

In particular, these test functions have a special role in the problem under study because the boundary value $v_{K}$ of an analytic function $\mathbf{v}_{K}$ on them can be represented in the form

$$
v_{K}(f)=\int \mathbf{v}_{K}(x+i y) f(x+i y) \mathrm{d} x \quad(y \in V,|y|<1 / B),
$$

which is convenient for analysis. Indeed, by the Cauchy-Poincaré theorem, the integral in the right-hand side does not depend on $y$ and coincides with the left-hand side by virtue of the relation $\lim _{y \rightarrow 0} f(x+i y)=f(x)$, which holds in the topology of $S_{a}^{1}$ and of course in that of $S$.

Theorem 1. Let $V$ be an open convex cone, let $\left(v_{K}\right)_{K \in I}$ be a countable family of tempered distributions serving as boundary values of functions $\mathbf{v}_{K}(z)$ holomorphic in $T^{V}=\{z: \operatorname{Im} z \in V\}$ and satisfying (23), and let $a$ and $b$ be the indicator functions of the space $S_{a}^{b}$ with the nontrivial subspace $S_{a}^{1}$. If there is a vector $\eta \in V$ such that

$$
\sum_{K \in I} \inf _{0<t<\delta} e^{s t} \int \frac{\left|\mathbf{v}_{K}(x+i t \eta)\right|}{a(|x| / A)} \mathrm{d} x \leq C_{\delta, \epsilon, A} b(\epsilon s)
$$

for any positive $\delta, \epsilon$ and $A$, then the family $\left(v_{K}\right)_{K \in I}$ is unconditionally summable in $S_{a}^{\prime b}$

Proof. Let $f \in S_{a, A}^{1, B}$ and $|\eta|=1$. Consecutively applying the representation (25), the definition (24), and the inequalities (26) with $s=0$ and $\delta<1 / B$ yields

$$
\sum_{K \in I}\left|v_{K}(f)\right| \leq\|f\|_{A, B} \sum_{K \in I} \inf _{0<t<\delta} \int \frac{\left|\mathbf{v}_{K}(x+i t \eta)\right|}{a(|x| / A)} \mathrm{d} x \leq C_{A, B} .
$$

Therefore, the family of distributions $v_{K}$ is absolutely summable on each element of $S_{a}^{1}$. Because this is a Montel space, its strong dual is also a Montel space, and it contains an element $v$ to which the family in question is unconditionally summable in the strong topology. We investigate the convolution of the Fourier transform $\hat{v}=u \in S_{1}^{\prime a}$ and a test function $g \in S_{1, B}^{a, A}$ using the relation

$$
(u * g)(p)=\sum_{K \in I} \int \mathbf{v}_{K}(x+i y) e^{i p(x+i y)} f(x+i y) \mathrm{d} x,
$$


which holds for any $y$ belonging to both the domain $V$ and the base of the analyticity domain of the function

$$
f(z)=\int g(-p) e^{i p z} \mathrm{~d} p
$$

The Fourier operator realizes a one-to-one bicontinuous mapping of $S_{1}^{a}$ onto $S_{a}^{1}$, and

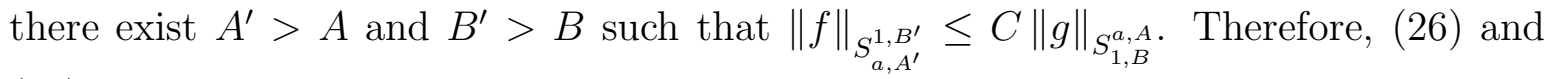
(27) imply the estimate

$$
|(u * g)(p)| \leq C\|g\|_{A, B} \sum_{K \in I} \inf _{0<t<1 / B^{\prime}} e^{|p| t} \int \frac{\left|\mathbf{v}_{K}(x+i t \eta)\right|}{a\left(|x| / A^{\prime}\right)} \mathrm{d} x \leq C_{\epsilon, A}\|g\|_{A, B} b(\epsilon|p|),
$$

whence $u \in S_{b}^{\prime a}$ by Lemma 3 .

Now let $f \in S_{a}^{b}, f_{\mu} \in S_{a}^{1}$, and $f_{\mu} \rightarrow f$ in $S_{a}^{b}$. We fix a one-to-one mapping $\mathbb{N} \rightarrow I$ : $\nu \rightarrow K_{\nu}$ and write

$$
v_{\lambda}(f)=\sum_{\nu \leq \lambda} v_{K_{\nu}}(f)
$$

and

$$
v(f)-v_{\lambda}(f)=v\left(f-f_{\mu}\right)+\left[v\left(f_{\mu}\right)-v_{\lambda}\left(f_{\mu}\right)\right]+v_{\lambda}\left(f_{\mu}-f\right)
$$

The Fourier transform of each $v_{\lambda}$ satisfies the inequality of the form (28) with the same constant $C_{\epsilon, A}$ for all $\lambda$. Consequently, by Lemma 3, this sequence of distributions is bounded in $S_{a}^{\prime b}$. As in the case of an arbitrary reflexive space, the topology of $S_{a}^{b}$ coincides with the uniform convergence topology on bounded sets. The limiting relation $v_{\lambda}\left(f_{\mu}-f\right) \rightarrow 0$ therefore holds uniformly with respect to $\lambda$ as $\mu \rightarrow \infty$. For a fixed sufficiently large $\mu$ and an arbitrary $\varepsilon>0$, there is a number $\lambda_{\varepsilon, \mu}$ such that $\left|v\left(f_{\mu}\right)-v_{\lambda}\left(f_{\mu}\right)\right|<\varepsilon$ for $\lambda>\lambda_{\varepsilon, \mu}$. We therefore have $v_{\lambda} \rightarrow v$ in the weak and consequently in the strong topologies of the Montel space $S_{a}^{\prime b}$. The theorem is proved.

Wick power series converging to nonlocal fields [2] are of interest in field theory with positive metric. An analogue of Theorem 1 is useful in their analysis. The role of $S_{a}^{1}$ in its derivation is played by the space $S^{0}(\beta=0)$, which is the Fourier transform of the space $S_{0}=C_{0}^{\infty}$ of smooth functions with compact support. More precisely, $S^{0}=\bigcup_{B>0} S^{0, B}$, where $S^{0, B}$ consists of the entire functions $f(z)$ such that the norm

$$
\|f\|_{B, N}=\sup _{z=x+i y}(1+|z|)^{N}|f(z)| e^{-B|y|}
$$

is finite for any $N$. 
Theorem 2. As in Theorem 1 , let $\left(v_{K}\right)_{K \in I}$ be a countable family of tempered distributions serving as boundary values of the functions $\mathbf{v}_{K}(z)$ holomorphic in the domain $T^{V}$ and satisfying the condition (23). Let $b$ be the indicator function of the space $S^{b}$. If there is a vector $\eta \in V$ such that

$$
\sum_{K \in I} \inf _{t>0} e^{s t} \int \frac{\left|\mathbf{v}_{K}(x+i t \eta)\right|}{(1+|x|)^{N}} \mathrm{~d} x \leq C_{\epsilon} b(\epsilon s),
$$

for any $\epsilon>0$ and some $N(\epsilon)$, then the family $\left(v_{K}\right)_{K \in I}$ is unconditionally summable in $S^{\prime b}$.

Proof. Let $f \in S^{0, B}$ and $|\eta|=1$. Then

$$
|f(x+i t \eta)| \leq\|f\|_{B, N} e^{B t}(1+|x|)^{-N} .
$$

In view of (25) and (29) with $s=B$, we conclude that the family of distributions $v_{K}$ is absolutely summable on every element of $S^{0}$. We let $u$ denote the Fourier transform of the limit and consider the convolution of $u$ with a function $g$ in $S_{0, B}$. By the definition of $S_{0, B}$, the support of $g$ lies in the ball $|p|<B$, and all the norms $\|g\|_{S_{0, B, N}}=\sup _{|p| \leq B} \max _{|\kappa| \leq N}\left|\partial^{\kappa} g(p)\right|$ are finite. Let $f(z)$ be defined as in the proof of Theorem 1. For a sufficiently large $B^{\prime}$, we have a continuous embedding $S_{0, B} \rightarrow S^{0, B^{\prime}}$, and for any $N^{\prime}$ there exists an $N$ such that

$$
\|f\|_{S^{0, B^{\prime}, N^{\prime}}} \leq C\|g\|_{S_{0, B, N}} .
$$

Let $C_{\epsilon}$ and $N^{\prime}(\epsilon)$ be the numbers involved in (29). Applying (27), (30), (29), and (17) consecutively, we obtain

$$
|(u * g)(p)| \leq C C_{\epsilon}\|g\|_{B, N(\epsilon)} b\left(2 B^{\prime} \epsilon\right) b(2 \epsilon|p|)
$$

We now apply Lemma 3 to $u$ and to the family $u_{\lambda}$ of the Fourier transforms of partial sums $v_{\lambda},(\lambda \in \mathbb{N})$, to conclude that $v \in S^{\prime b}$ and that the sequence $v_{\lambda}$ is bounded in $S^{\prime b}$. The remaining part of the proof coincides with the end of the proof of Theorem 1 because $S^{b}$ are also Montel spaces.

\section{$5 \quad$ Test function space for Wick series}

When studying the behavior of analytic functions whose boundary values are distributions (14), we can replace all functions $\mathbf{w}\left(z_{j}-z_{m}\right)$ with $\mathbf{w}_{\text {maj }}\left(z_{j}, z_{m}\right)$ because

$$
\left|\mathbf{w}\left(x-x^{\prime}-2 i y\right)\right|^{2} \leq\left|\mathbf{w}_{\text {maj }}(x-i y, x+i y)\right|\left|\mathbf{w}_{\text {maj }}\left(x^{\prime}-i y, x^{\prime}+i y\right)\right|
$$


for all $y \in \mathbb{V}_{+}$. Indeed, (2) implies the inequality

$$
\left|\left\langle\phi(f) \Psi_{0}, \phi(g) \Psi_{0}\right\rangle\right| \leq\left\|\phi(f) \Psi_{0}\right\|\left\|\phi(g) \Psi_{0}\right\|
$$

Taking $f(\xi)=(\nu / \sqrt{\pi})^{\mathrm{d}} e^{-\nu^{2}(\xi-x-i y)^{2}}$ and $g(\xi)=(\nu / \sqrt{\pi})^{\mathrm{d}} e^{-\nu^{2}\left(\xi-x^{\prime}-i y\right)^{2}}$ and writing the left- and right-hand sides in this inequality as integrals over a plane in the analyticity domain and passing to the limit as $\nu \rightarrow \infty$, we immediately obtain (31). Everywhere below, we assume that the imaginary parts of the first and second arguments of the function $\mathbf{w}_{\text {maj }}$ belong to the respective negative and positive half-lines $y_{0}$ and characterize its infrared and ultraviolet behavior with a pair of monotonic nonnegative functions $w_{I R}$ and $w_{U V}$. The first (second) function increases as the argument increases (as the argument decreases), and the inequality

$$
\left|\mathbf{w}_{\text {maj }}\left(z, z^{\prime}\right)\right| \leq C_{0}+C_{1} w_{I R}\left(|z|+\left|z^{\prime}\right|\right)+C_{2} w_{U V}\left(|y|+\left|y^{\prime}\right|\right) .
$$

is assumed. By formula (5), such characteristic functions $w_{I R}$ and $w_{U V}$ exist and can be easily chosen in each specific case, particularly with preservation of the normalization condition $w_{I R}(0)=w_{U V}(\infty)=0$.

We seek the test function space for the series (1) under some natural conditions on the coefficients of the series. We assume that

$$
d_{k} \geq 0, \quad d_{0}=1, \quad \lim _{k \rightarrow \infty}\left(k ! d_{2 k}\right)^{1 / k}=0 .
$$

The first two conditions are purely technical and are justified because every series is subordinate to a series satisfying these conditions. In a theory with a positive metric, the third condition ensures that the sum of the series is a local field, and in the case of an indefinite metric, it is necessary for the very existence of the sum. Moreover, we assume that

$$
d_{k} d_{l} \leq C h^{k+l} d_{k+l}
$$

where $C$ and $h$ are some constants. In this case, the restrictions on the test functions under which the complete operator realization of the field (1) is possible coincide essentially with those guaranteeing the convergence of this series on the vacuum vector. If the conditions (33) and (34) hold, then $d_{k}^{2} \leq C h^{2 k} d_{2 k}$, and the two-point function

$$
\left\langle\Psi_{0}, \varphi\left(x_{1}\right) \varphi\left(x_{2}\right) \Psi_{0}\right\rangle=\sum_{k} k ! d_{k}^{2} w\left(x_{1}-x_{2}\right)^{k}
$$


is analytic in a domain ordinary for the local theory because it is the composition of $w$ and some entire function. Similarly, the function $\sum_{k} k ! d_{k}^{2} w_{\text {maj }}\left(x_{1}, x_{2}\right)^{k}$ entering (9) inherits the analytic properties of $w_{\text {maj }}$.

Theorem 3. Let $\phi$ be a free field acting in the pseudo-Hilbert space $\mathcal{H}$, and let the positive majorant of its correlation function satisfy the inequality (32) in which $w_{I R}$ and $w_{U V}$ are monotonic. Under the conditions (33) and (34) on the coefficients, the series $\varphi=\mathfrak{s}(\phi)$ in the Wick powers of this field and also any of its subordinate series are well defined as operator-valued generalized functions on every space $S_{a}^{b}$ containing a nontrivial subspace $S_{a}^{1}$ whose indicator functions satisfy the inequalities

$$
\sum_{k} L^{k} k ! d_{2 k} w_{I R}(r)^{k} \leq C_{L, \epsilon} a(\epsilon r), \quad \inf _{t>0} e^{s \tau} \sum_{k} L^{k} k ! d_{2 k} w_{U V}(t)^{k} \leq C_{L, \epsilon} b(\epsilon s) .
$$

for an arbitrarily large $L>0$ and an arbitrarily small $\epsilon>0$.

Proof. We set $v_{K}=D_{K} W^{K}$ and apply Theorem 1 to the series (13). Let $e_{0}$ be the unit vector $(1,0)$ in $\mathbb{R}^{d}$, let $\eta$ be a vector in $\mathbb{R}^{2 n d}$ with the components $\eta_{j}=-j e_{0}$ for $1 \leq j \leq n$ and $\eta_{j}=(j-n) e_{0}$ for $n+1 \leq j \leq 2 n$, and let $r=|x|$, where $x=\left(x_{1}, \ldots, x_{2 n}\right)$. Applying (31) and (32) in view of the monotonicity of the functions $w_{I R}$ and $w_{U V}$ yields

$$
\left|\mathbf{W}^{K}(x+i t \eta)\right| \leq 3^{|K|-1}\left(C_{0}^{|K|}+C_{1}^{|K|} w_{I R}(2 r+2 n t)^{|K|}+C_{2}^{|K|} w_{U V}(t)^{|K|}\right) .
$$

The condition (34) and the inequalities $|K| ! / K ! \leq(n(2 n-1))^{|K|}$ and $\kappa ! \leq|\kappa| ! \leq$ $4^{|K|}(|K| !)^{2}$ following from the well-known properties of polynomial coefficients imply

$$
D_{K} \leq C^{\prime} h^{\prime|K|}|K| ! d_{2|K|}
$$

where the constant $h^{\prime}$ depends on $n$. The summation over the multi-indices can now be replaced with that over the positive integers. The number of multi-indices with a given norm $|K|$ is equal to the binomial coefficient $\left(\begin{array}{c}|K|+n(2 n-1)-1 \\ |K|\end{array}\right)$. This dependence on $|K|$ is polynomial and therefore insignificant. Using (36) and (37), we obtain

$$
\sum_{K}\left|\mathbf{v}_{K}(x+i t \eta)\right| \leq C^{\prime \prime}\left(\sum_{k=0}^{\infty} L^{k} k ! d_{2 k} w_{I R}(2 r+2 n t)^{k}\right)\left(\sum_{k=0}^{\infty} L^{k} k ! d_{2 k} w_{U V}(t)^{k}\right) .
$$

The first of the conditions (35) and the analogue of the inequality (17) for the indicator function $a$ permits majorizing the sum of the powers of $w_{I R}$ with the expression $C_{L, \epsilon}(t) a(4 \epsilon r)$, where $C_{L, \epsilon}(t)$ is a bounded function on every finite interval. Applying the 
condition (18) to $a$ results in $(1+r)^{N} a(4 \epsilon r) \leq C a(r / A)$ if an $\epsilon<1 /\left(4 A \lambda^{N}\right)$ is chosen. Taking $N=2 n \mathrm{~d}+1$, we obtain the estimate

$$
\sum_{K} \int \frac{\left|\mathbf{v}_{K}(x+i t \eta)\right|}{a(|x| / A)} \mathrm{d} x \leq C_{A, L}(t) \sum_{k=0}^{\infty} L^{k} k ! d_{2 k} w_{U V}(t)^{k} .
$$

Because $w_{U V}$ is monotonically increasing with a decreasing argument, the infimum in the second formula in (35) occurs on the interval $(0, \delta)$ for sufficiently large $s$, where $\delta$ is arbitrarily small. Since the sum of the infima does not exceed the infimum of the sum, we conclude that (35) implies (26). By Theorem 1, the series $\sum_{K} D_{K} W^{K}$ is unconditionally summable in $S_{a}^{\prime b}$, and the number series (13) is absolutely summable for any test function $f \in S_{a}^{b}$. To complete the proof, it remains to apply Lemmas 1 and 2 .

Remark. The temperedness of growth means that the singularities of $w_{\text {maj }}$ are no worse than polynomial or logarithmic ones. Therefore, it can be assumed that the inequalities

$$
w_{I R}(\lambda r) \leq C_{\lambda} w_{I R}(r), \quad w_{U V}(t / \lambda) \leq C_{\lambda}^{\prime} w_{U V}(t),
$$

hold for any $\lambda>0$, at least in the limiting sense, i.e., for $r>R(\lambda)$ and $t<\delta(\lambda)$. In this case, the criterion (35) takes a simpler form

$$
\sum_{k} L^{k} k ! d_{2 k} w_{I R}(r)^{k} \leq C_{L} a(r), \quad \inf _{t>0} e^{s t} \sum_{k} L^{k} k ! d_{2 k} w_{U V}(t)^{k} \leq C_{L} b(s),
$$

where $L>0$ is arbitrarily large. In particular, the conditions (38) when applied to the normal exponential : $\exp i g \phi:(x)$ become

$$
\exp \left\{L w_{I R}(r)\right\} \leq C_{L} a(r), \quad \inf _{t>0} \exp \left\{s t+L w_{U V}(t)\right\} \leq C_{L} b(s) .
$$

\section{The case of a positive metric}

We now apply the suggested construction to the simplest case of a free scalar field with the Wightman function

$$
\Delta^{+}(x ; m)=\frac{1}{(2 \pi)^{\mathrm{d}-1}} \int \theta\left(p^{0}\right) \delta\left(p^{2}-m^{2}\right) e^{-i p x} \mathrm{~d} p=\frac{1}{(2 \pi)^{\mathrm{d}-1}} \int \frac{e^{-i \omega(\mathbf{p}) x^{0}}}{2 \omega(\mathbf{p})} e^{i \mathbf{p x}} \mathrm{d} \mathbf{p} .
$$

Let the dimension of the space-time be $d>2$. Using the inequality $\omega(\mathbf{p})=\sqrt{\mathbf{p}^{2}+m^{2}} \geq$ $\varepsilon|\mathbf{p}|+m \sqrt{1-\varepsilon^{2}}$ and the notation $s=|\mathbf{p}|, m^{\prime}=m \sqrt{1-\varepsilon^{2}}$, we derive the elementary 
estimate

$$
\left|\Delta^{+}\left(x^{0}-i t, \mathbf{x} ; m\right)\right| \leq C e^{-m^{\prime} t} \int_{0}^{\infty} e^{-\varepsilon s t} s^{\mathrm{d}-3} \mathrm{~d} s=C^{\prime} e^{-m^{\prime} t} / t^{(\mathrm{d}-2)}
$$

showing that $w_{I R}=0$ and $w_{U V}(t)=e^{-m^{\prime} t} / t^{(\mathrm{d}-2)}$ can be taken in this case. In view of the infrared boundedness of the distribution $\Delta^{+}$, we can take $S^{0}$ as the initial test function space for the Wick series. For the same reason, Wick series can be convergent even when the localization condition (33) is violated. Theorem 2 permits analyzing this situation and strengthening Rieckers' results [2] (see the following theorem).

Theorem 4. Let $\phi$ be a free neutral scalar field of mass $m>0$ in a space-time of dimension $\mathrm{d}>2$. The Wick series $\sum_{k=0}^{\infty} d_{k}: \phi^{k}:(x)$ with positive coefficients satisfying the condition (34) is well defined in the Fock space of the field $\phi$ under smoothing with test functions in the space $S^{b}$ for which

$$
\sum_{k<s} k ! d_{2 k}(s / k)^{k(\mathrm{~d}-2)} \leq C_{\epsilon} b(\epsilon s)
$$

with an arbitrary $\epsilon>0$. If $m=0$, then this series is well defined under the stronger condition

$$
\sum_{k=0}^{\infty} k ! d_{2 k}(s / k)^{k(\mathrm{~d}-2)} \leq C_{\epsilon} b(\epsilon s)
$$

Proof. The theorem can be proved by repeating the proof of Theorem 3 with obvious changes of some details. The main distinction is that in Theorem 3 we first estimated the sum of analytic functions and only then took the infimum over $t$, whereas here we choose a free parameter in the representation (25) for each individual term of the series. Using the same notation, applying (36) and (37), and setting $N=2 n \mathrm{~d}+1$, we obtain

$$
\sum_{K} \inf _{t>0} e^{s t} \int \frac{\left|\mathbf{v}_{K}(x+i t \eta)\right|}{(1+|x|)^{N}} \mathrm{~d} x \leq C \sum_{k=0}^{\infty} L^{k} k ! d_{2 k} \inf _{t>0} \frac{e^{\left(s-k m^{\prime}\right) t}}{t^{k(\mathrm{~d}-2)}} .
$$

Let $m \neq 0$. We write $\lambda=e /(\mathrm{d}-2)$. The infimum in (41) is equal to zero for $k \geq s / m^{\prime}$ and is equal to $\left(\lambda\left(s / k-m^{\prime}\right)\right)^{k(\mathrm{~d}-2)} \leq(\lambda s / k)^{k(\mathrm{~d}-2)}$ for other values of $k$. We assume that $\lambda^{\prime}=L^{1 /(\mathrm{d}-2)} \lambda \geq 1 / m^{\prime}$. It then follows from (39) that the right-hand side of (41) is majorized by $C_{\epsilon} b\left(\epsilon \lambda^{\prime} s\right)$, which ensures the fulfillment of the conditions of the Theorem 2 because $\epsilon$ is arbitrary. For $m=0$, no truncation of summation occurs, and the same result is ensured by the formula (40). Applying Lemmas 1 and 2 completes the proof. 
We note that in the localizable case, the variation of the plane of integration in each individual term of the series gives no significant improvement of the estimate and the conditions (39) and (40) become equivalent. Indeed, let the coefficients $d_{k}$ have the form

$$
d_{k}=k !^{-1 / \rho}, \quad \rho>0 .
$$

Simple calculations using the Stirling formula that are similar to those in Sec.IV.2.2 in [8] show that the functions

$$
\inf _{t>0} e^{s t} \sum_{k=0}^{\infty} k ! d_{2 k} / t^{k(\mathrm{~d}-2)}
$$

and

$$
\sum_{k=0}^{\infty} k ! d_{2 k} \inf _{t>0} e^{s t} / t^{k(\mathrm{~d}-2)}=\sum_{k=0}^{\infty} k ! d_{2 k}(e s / k(\mathrm{~d}-2))^{k(\mathrm{~d}-2)}
$$

have the same order of growth $\varrho=(\mathrm{d}-2) /(\mathrm{d}-3+2 / \rho)$ for $\rho<2$ and differ only in their types. The maximum term in the second of these series corresponds to the index $k(s) \sim s^{\varrho}$, and the restriction of the summation to the range $k<s$ therefore does not affect the asymptotic behavior for $\varrho<1$, which is equivalent to $\rho<2$. In contrast, for $\rho>2$, the truncated series behaves as $e^{(1-2 / \rho) s \ln s}$ as $s \rightarrow \infty$. In particular, all entire functions of a massive field with a finite order of growth are well defined on any of the spaces $S^{\beta}$ with the index $\beta<1$ and even on $S^{b}$, where $b(s)=s(\ln (1+s))^{\gamma}, \gamma>1$, whereas the universal space for realizing finite-order entire functions in the massless case is the narrower space $S^{1-1 /(\mathrm{d}-2)}$.

\section{$7 \quad$ Wick series for the two-dimensional massless field}

We consider the simplest example of a field with singular infrared behavior. Quantizing such a field requires introducing an indefinite metric (see [3]). Pierotti constructed the normal exponential of this field with an exact description of the test function space [4]. Here, we describe suitable test functions for realizing the Wick entire functions of this field that have an exponential growth with the order $\rho<2$. For $\mathrm{d}=2$, the singular expression $2 \pi \theta\left(p^{0}\right) \delta\left(p^{2}\right)$ has a clear meaning on the subspace $S_{\bullet} \subset S$ consisting of the functions vanishing at $p=0$. It is written as $\pi\left(\theta(u) u^{-1} \delta(v)+\theta(v) v^{-1} \delta(u)\right)$ in the light cone variables $u=p_{0}+p_{1}$ and $v=p_{0}-p_{1}$. Its Lorentz invariant extension to $S$ has 
the form

$$
\pi\left(u_{+}^{-1} \delta(v)+v_{+}^{-1} \delta(u)\right)+c \delta(u) \delta(v)
$$

where

$$
u_{+}^{-1}(f) \stackrel{\text { def }}{=}-\int_{0}^{\infty} f^{\prime}(u) \ln u \mathrm{~d} u,
$$

and is not positively definite for any value of the constant $c$. Passing to Laplace transforms, we obtain

$$
w(z)=-\frac{1}{4 \pi} \ln \left(-\kappa^{2} z^{2}\right) \quad\left(\operatorname{Im} z \in \mathbb{V}_{-}\right),
$$

where $\kappa$ is an arbitrary scaling parameter whose exact relation to the constant $c$ in (143) is insignificant. Let $h \in S$ and $\hat{h}(0)=1$. Then $\hat{f}_{0}=\hat{f}-\hat{f}(0) \hat{h} \in S$. We write

$$
\langle f, g\rangle=\int \bar{f}(x) w\left(x-x^{\prime}\right) g(x) \mathrm{d} x \mathrm{~d} x^{\prime}
$$

and set $\langle h, h\rangle=0$, which can be ensured by an appropriate scale transformation. In this case, we have

$$
\langle f, g\rangle=\left\langle f_{0}, g_{0}\right\rangle+\overline{\hat{f}}(0)\langle h, g\rangle+\hat{g}(0)\langle f, h\rangle .
$$

According to [1, 10], $w_{\text {maj }}$ is given by the relation

$$
\int \bar{f}(x) w_{\text {maj }}\left(x, x^{\prime}\right) g\left(x^{\prime}\right) \mathrm{d} x \mathrm{~d} x^{\prime}=\left\langle f_{0}, g_{0}\right\rangle+\langle f, h\rangle\langle h, g\rangle+\overline{\hat{f}}(0) \hat{g}(0) .
$$

If $h$ is a real-valued even function, then the explicit form of the majorant is

$$
w_{\text {maj }}\left(x, x^{\prime}\right)=w\left(x-x^{\prime}\right)+\left(w_{h}(x)-1\right)\left(w_{h}(-x)-1\right),
$$

where $w_{h}=w * h$. Let $\operatorname{Im} z=-t e_{0}, e_{0}=(1,0)$ and $t>0$. Then $t^{2} \leq\left|z^{2}\right|$. On the other hand, $\left|z^{2}\right| \leq 2|z|^{2}$, and consequently $\left|\ln \left(-z^{2}\right)\right| \leq C+2 \ln (1+|z|)+2 \ln ^{+}(1 / t)$, where $\ln ^{+}(\cdot)=\max (0, \ln (\cdot))$. Let $h \in S^{0}$. Then $w_{h}$ is an entire function satisfying the inequality $\left|w_{h}(z)\right| \leq C+C^{\prime} \ln (1+|z|)$ for the indicated values of $\operatorname{Im} z$. It can be easily established using the representation

$$
w_{h}(z)=\int h\left(\xi+i e_{0}\right) w(z-\xi-i e) \mathrm{d} \xi
$$

and the inequality $1+|z-\xi| \leq(1+|z|)(1+|\xi|)$. We can therefore take

$$
w_{I R}(r)=(\ln (1+r))^{2}, \quad w_{U V}(t)=\ln ^{+}(1 / t) .
$$


in the case under consideration. In this case, the role of the test function space for realizing any Wick entire function of order $\rho<2$ can clearly be played by $S_{\alpha}^{\beta}$ with arbitrarily large $\alpha$ and $\beta$. It is also easy to obtain a more exact description. For the coefficients $d_{k}$ of the form (42), the inequalities

$$
\sum_{k} L^{k} k ! d_{2 k} w_{I R}(r)^{k} \leq C e^{N(\ln (1+r))^{2 \rho /(2-\rho)}}, \inf _{t>0} e^{s t} \sum_{k} L^{k} k ! d_{2 k} w_{U V}(t)^{k} \leq C e^{N(\ln (1+s))^{\rho /(2-\rho)}}
$$

hold, where $N$ depends on $L$ and the infimum can be estimated by the value of the function at the point $t=1 / s$, which does not in fact lead to cruder results. In particular, if $\rho \leq 2 / 3$, then the conditions (35) hold for $a(r)=(1+r)^{N}$ and $b(s)=1+s$. Hence, for this order of growth, the operator realization with test functions in the Schwartz space $S$ is possible, which can be seen if $S^{0}$ is taken as the original test function space and an analogue of Lemma 3 for the simplest case of the extension of $u \in S_{0}^{\prime}$ to $S$ is used. Namely, such an extension is sure to exist, if $|(u * g)(p)| \leq C_{B}\|g\|_{B, N}(1+|p|)^{N}$ for some $B$ and $N$. For $2 / 3<\rho \leq 1$, the behavior is polynomially bounded with respect to $s$ as before but is no longer polynomially bounded with respect to $r$, namely it is characterized in the latter case by the function $e^{(\ln (1+r))^{\alpha}}$, where $\alpha=2 \rho /(2-\rho)$. If $\alpha<2$ (i.e., $\rho<1$ ), then the function with such a slow growth cannot serve as an indicator function because it does not satisfy the condition (18). The space $S_{a}$, where $a=e^{(\ln (1+r))^{2}}$, is already acceptable and can serve as the test function space for any $\rho<1$ by Theorem 2. For $\rho=1$ and a finite order of growth, the series (13) converges on the functions in $S_{a_{N}}$, where $a_{N}=e^{N(\ln (1+r))^{2}}$ and $N$ grows with increasing $n$. Therefore, the adequate space for this case is $\mathcal{P}_{2}=\bigcap_{N} S_{a_{N}}$. It consists of smooth functions such that the norms

$$
\|f\|_{N}=\sup _{x} \max _{|\kappa| \leq N}\left|\partial^{\kappa} f(x)\right| e^{N(\ln (1+r))^{2}} \quad(N=0,1,2, \ldots) .
$$

are finite and belongs to the class $K\left(M_{N}\right)$ (see [8] for the theory of these spaces). For $\rho>1$, it is natural to use the spaces

$$
\mathcal{P}_{\alpha}^{\beta}=\bigcap_{N=0}^{\infty} S_{a_{\alpha, N}}^{b_{\beta, N}},
$$

where

$$
a_{\alpha, N}(r)=e^{N(\ln (1+r))^{\alpha}}, \quad b_{\beta, N}(s)=e^{N(\ln (1+s))^{\beta}}, \quad \alpha, \beta \geq 2 .
$$


Ultimately, we obtain the following result.

Theorem 5. Let $\phi$ be a free massless scalar field in a space-time of dimension 2. The role of the test function space for its normally ordered entire functions of the order $\rho<2$ and of finite type can be played by the following spaces:

the Schwartz space $S$ for $\rho \leq 2 / 3$;

the space $\mathcal{P}_{2}$ for all functions of the exponential type;

the space $\mathcal{P}_{2 \rho /(2-\rho)}^{2}$ for $1<\rho \leq 4 / 3$;

the space $\mathcal{P}_{2 \rho /(2-\rho)}^{\rho /(2-\rho)}$ for $4 / 3<\rho<2$.

\section{Conclusion}

The presented theorems reduce finding the test function space for normally ordered power series of a free field to estimating the order of growth and the order of singularity for the Hilbert majorant of the two-point function of the field. If the adequate test function space contains functions of compact support in both the coordinate and momentum representations, then the limit of the Wick series satisfies the main general requirements of quantum field theory [11] in a practically obvious manner because we have constructed the operator realization in the very state space of the original free field. However, as a rule, the localizability condition does not hold in momentum space for the exact solutions of gauge models in a generic covariant gauge [5, 6], and the Wick series involved in them are defined only on test functions whose Fourier transforms are analytic. The statement of the spectral condition and the verification of its fulfillment is a nontrivial problem in this case [6, 14]. Moreover, this problem is of particular interest because its solution is instructive for the correct generalization of the reconstruction theorem and the Osterwalder-Schrader Euclidean theory to quantum field theory with infrared singular indefinite metric, where the Poincaré group is implemented by pseudounitary operators. We plan to investigate this circle of problems in a separate publication. Another interesting problem whose solution can be obtained by applying the approach developed in this paper is the problem of constructing the nonlocal extension of the Borchers equivalence classes and proving that the nonlocal Wick series of a free field with a positive metric satisfy the condition of asymptotic commutativity [15] which ensures the preservation of the main physical consequences 
in nonlocal quantum field theory.

Acknowledgments. This work is supported by the Russian Foundation for Basic Research (Grant No. 99-01-00376) and INTAS (Grant No. 96-0308).

[1] A. Jaffe. Ann. Phys. 1965. V. 32. P. 127.

[2] A. Rieckers. Int. J. Theor. Phys. 1971. V. 4. P. 55.

[3] A. S. Wightman. Adv. Math. Suppl. Stud. 1981. V. 7B. P. 769.

[4] D. Pierotti. Lett. Math. Phys. 1988. V. 15. P. 219.

[5] U. Moschella and F. Strocchi. Lett. Math. Phys. 1992. V. 24. P. 103.

[6] U. Moschella. J. Math. Phys. 1993. V. 34. P. 535.

[7] M. A. Soloviev. Lett. Math. Phys. 1997. V. 41. P. 265.

[8] I. M. Gelfand and G. E. Shilov. Generalized Functions. Vol. 2. New York: Academic Press, 1968.

[9] M. A. Soloviev. Beyond the theory of hyperfunctions. In: Developments in Mathematics: The Moscow School, V. Arnold and M. Monastyrsky (eds.). London: Chapman and Hall, 1993. P. 131.

[10] G. Morchio, F. Strocchi. Ann. Inst. Henri Poincaré. 1980. V. 33A. P. 251.

[11] N. N. Bogolyubov, A. A. Logunov, A. I. Oksak, I. T. Todorov. General Principles of Quantum Field Theory. Dordrecht: Kluwer, 1990.

[12] M. Reed, B. Simon. Methods of Modern Mathematical Physics. Vol 1. New York, London: Academic Press, 1972.

[13] H. H. Schaefer. Topological Vector Spaces. New York, Heidelberg, Berlin: SpringerVerlag, 1970. 
[14] M. A. Soloviev. Theor. Math. Phys. 1995. V. 105. P. 1520.

[15] M. A. Soloviev. Theor. Math. Phys. 1999. V. 121. P. 1377. 